\title{
Y-Ca-In-GelG 的离子分布和磁性
}

\author{
林梅梅 \\ 李 \pm \\ 李国标 \\ （清华大学工程物理系）（中国科学院高能物理研究所）（中国科学院物理研究所）
}

钎-钙-钢-锗石榴石型铁氧体 (Y-Ca-In-GeIG) 是一种饱和磁化强度较低, 铁磁共振线宽 很窄的微波铁氧体, 它是从钎石榴石型铁氧体（YIG）经离子代换而得到的, 其磁性与阳离子 在四面体 (d)、八面体 (a) 和十二面体 (c) 晶位上的分布有密切的联系, 因而受到基础研究和 实际应用两方面的重视. 本文从穆斯堡尔效应研究了 $\mathrm{Fe}$ 离子的分布, 并由此探讨了离子分布 与磁性的关系.

利用 $\mathrm{Ca}$ 和 $\mathrm{In} 、 \mathrm{Ge}$ 分别部分代换 $\mathrm{YIG}$ 中的 $\mathrm{Y}$ 和 $\mathrm{Fe}$ ，采取一般陶瓷工艺方法制备多晶铁氧 体,其化学分析结果为 $\mathrm{Y}_{2.78} \mathrm{Ca}_{0.25} \mathrm{In}_{0.60} \mathrm{Ge}_{0.25} \mathrm{Fe}_{4.11} \mathrm{O}_{12}$ (附录 1), 室温下的饱和磁化强度 $4 \pi M_{s}=1100$ 高斯, 铁磁共振线宽 $\Delta H \approx 2$ 奥(9.0 千兆赫), 1.8 奥(3.6 千兆赫), 居里点 $\theta_{f}=139^{\circ} \mathrm{C}^{[1]}$.

应用电磁驱动等加速度式穆斯堡尔谱仪 ${ }^{[2]}$ 在 $210^{\circ} \mathrm{C}$ 测量了样品的顺磁穆斯堡尔谱(图 1). 放射源为 ${ }^{7} \mathrm{Co}(\mathrm{Pd})$, 强度约 50 毫居里. 用 $\alpha-\mathrm{Fe}$ 的谱进行速度定标和确定零点. 利用电子 计算机对谱进行拟合. 这样得到两组顺磁谱, 分别相应于四面体 $(d)$ 和八面体 (a) 晶位的 $\mathrm{Fe}^{3+}$ 离子谱. 由于 YIG 中这两种晶位的 $\mathrm{Fe}^{3+}$ 离子无反冲分数几乎相等 ${ }^{[3]}$, 故可从谱线的面 积计算出 $d 、 a$ 晶位的 $\mathrm{Fe}^{3+}$ 离子数比 $N(d) / N(a)=2.1 \pm 0.1$. 由低温 (约 $80 \mathrm{~K}$ ) 铁磁谱的拟 合亦得到相近的结果, 但误差较大 ${ }^{[1]}$.

利用 MB-2 型磁天平测量了饱和磁矩 $\sigma_{s}$ 与温度 $T$ 的关系 (图 2). 外推到绝对零度的饱 和磁矩 $\sigma_{s}(0)=46 \pm 1$ 高斯. 厘米 ${ }^{3} /$ 克. 当知道 $\mathrm{Fe}^{3+}$ 离子在 $d$ 和 $a$ 晶位的分布后, 可以根

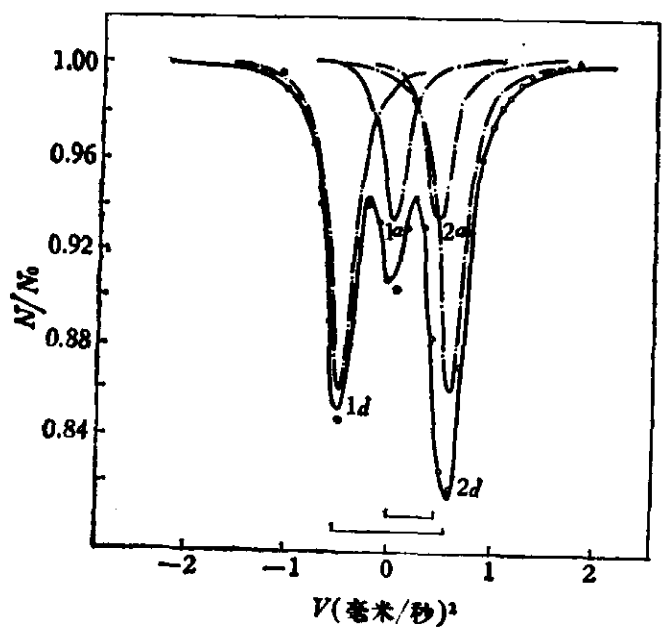

图 1 Y-Ca-In-GeIG 在 $210^{\circ} \mathrm{C}$ 的 穆斯堡尔谱 $(O)$ 及其拟合曲线

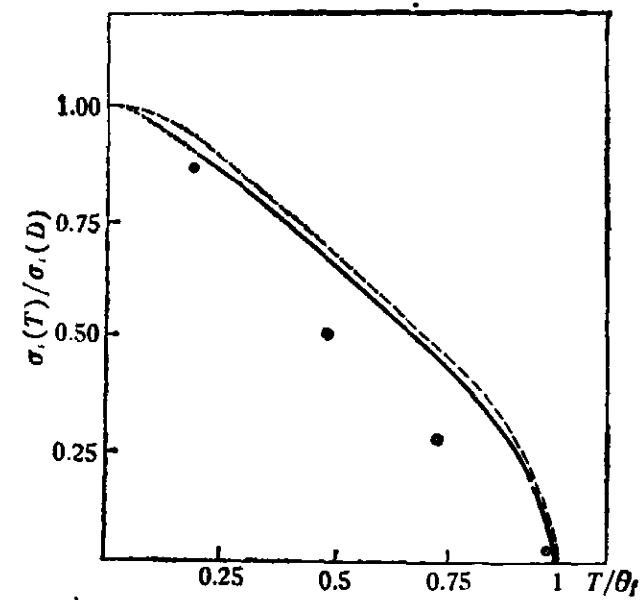

图 2 Y-Ca-In-GelG 的约化饱和磁矩 $\sigma_{s}(T) / \sigma_{s}(0)$ 与约化温度 $T / \theta_{f}$ 的关 系(实线), 以及根据 Néel 理论 $(\odot)$ 和 Dionne 理论(- - ) 的计算结果

本文 1981 年 5 月 21 日收到。 
表 I Y-Ca-In-GeIG 的绝对饱和磁矩 $\sigma_{s}(0)$ 的理论值与实验值的比较

\begin{tabular}{|c|c|c|c|c|c|c|}
\hline$N(d) / N(a)$ & $\begin{array}{c}\text { Néel } \\
\text { 理论值 }\end{array}$ & $\begin{array}{c}\text { Gilleo } \\
\text { 理论值 }\end{array}$ & $\begin{array}{c}\text { Geller } \\
\text { 理论值 }\end{array}$ & $\begin{array}{c}\text { Dionte } \\
\text { 理论值 }\end{array}$ & $\begin{array}{c}\text { 年正的 Dionne } \\
\text { 理论值 }\end{array}$ & 实验值 \\
\hline 2.0 & 49.9 & 40.4 & 45.8 & 46.8 & 46.0 & $46 \pm 1$ \\
\hline 2.1 & 52.9 & 41.5 & 48.6 & 49.5 & 48.7 \\
\hline 2.2 & 56.1 & 42.4 & 51.9 & 52.5 & 51.6 & \\
\hline
\end{tabular}

据”Nél 的简单亚铁磁性理论 ${ }^{[4]}$, Gilleo 的统计理 论 ${ }^{[5]}$, Geller $^{[6]}$ 和 Dionne ${ }^{[7]}$ 的无规成角亚铁 磁性理论计算出 $\sigma_{s}(0)$ 值 (表 1). 从表 1 中可以看出: Néel 和 Gilleo 理论值与实验值相 差较大, 而 Geller 和 Dionne 理论值与实验值较为接近, 但仍有差异. 作者将 Dionne 公式

$$
\begin{array}{r}
\sigma_{d}(0)=3 \times 5 \frac{N}{m} \mu_{B}\left(1-k_{d}\right)\left(1-0.1 k_{a}\right), \quad \sigma_{a}(0)=2 \times 5 \frac{N}{m} \mu_{B}\left(1-k_{a}\right)\left(1-k_{d}^{3.4}\right), \\
\sigma_{s}(0)=\sigma_{d}(0)-\sigma_{a}(0) \ldots \ldots \ldots \ldots
\end{array}
$$

中的系数 0.1 改为 0.124 后, 得到与实验值更为符合的结果. 上式中 $\sigma_{d}, \sigma_{a}$ 分别为 $d 、 a$ 晶位 的饱和磁矩, $N$ 为阿伏伽德罗数, $m$ 为克分子量, $k_{d} 、 k_{a}$ 分别为 $d 、 a$ 晶位的非磁性离子分数, $\mu_{B}$ 为玻尔磁子. 这是不难理解的. NEel 的简单亚铁磁性理论和 Gilleo 的超交换统计理论都 仅适用于非磁性离子代换量较小的情形，这时磁亚点阵间 $(d-a)$ 的交换作用占支配地位, 但 当非磁性离子代换量增大时, 磁亚点阵间的交换作用逐渐减弱, 因而必须考虑磁亚点阵内 $(d-d, a-a)$ 的交换作用, 即产生成角型亚铁磁性, 而不再是简单的共线型亚铁磁性. 因此, 考 虑了这一因素的 Geller 和 Dionne 理论便更接近于 Y-Ca-In-GeIG 的实际情况.

结合穆斯堡尔和饱和磁矩的实验结果, 以及离子半径 ${ }^{[8]}$ 和占位倾向 ${ }^{[0]}$ 的考虑, 可以看出, 与 实验符合最佳的在 $c\{\}, a\left[\right.$ 和 $d()$ 晶位的离子分布应该是: $\left\{\mathrm{Y}_{2.75} \mathrm{Ca}_{0.25}\right\}\left[\mathrm{Fe}_{1.36} \mathrm{I}_{0.6} \mathrm{Y}_{0.03}\right.$ ] $\left(\mathrm{Fe}_{2.75} \mathrm{Ge}_{0.25}\right) \mathrm{O}_{12}$, 其中值得注意的是约有 $1 \%$. $\mathrm{Y}^{3+}$ 离子进入八面体 $a_{\text {晶位 }}{ }^{[\text {特 } 2]}$. 这种情况 在这种类型的 YIG 材料中未见报道. 考 虑到在八面体 $(a)$ 晶位中, $\mathrm{Y}^{3+}$ 离子半径 $(0.892 \AA)^{[100]}$ 比 $\mathrm{In}^{3+}$ 离子半径 $(0.80)$ 还大, 因 而有可能对于降低磁晶各向异性场 ${ }^{[1]}$, 降低 多晶铁磁共振线宽起有益的作用. 当然, 此 结论尚需进一步的实验验证.

进一步考察非磁性离子代换对于饱和磁 矩随温度变化的影响. 根据 Néel 的亚铁磁 性理论, 可以从亚铁磁体的顺磁磁化率倒数温度 $(1 / \chi-T)$ 曲线的拟合 (图 3), 求出磁亚

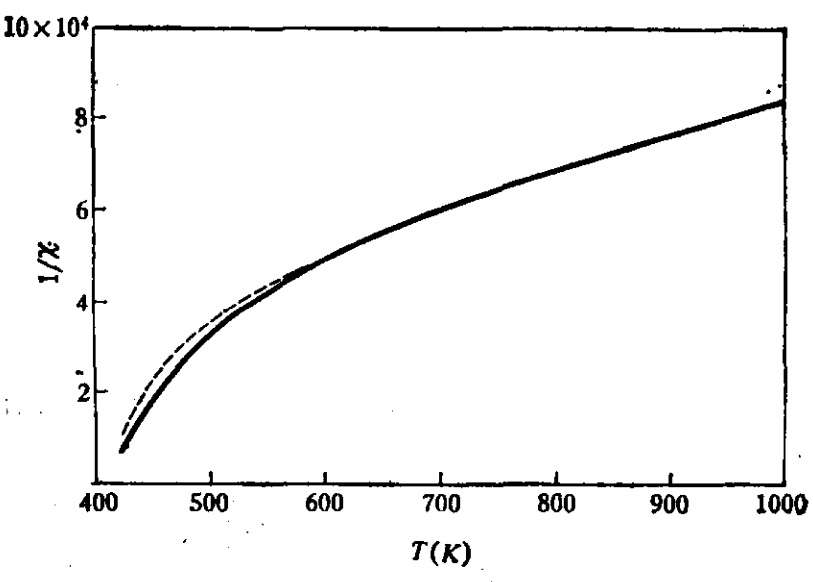

图 3 Y-Ca-In-GeIG 顺磁磁化率倒数 $1 / x$ 与温度 $T$ 的关系 (实线)，以及按 Néel 理论的拟合计算 曲线 (虚线)

点阵间和磁亚点阵内的分子场系数 ${ }^{[4,123}$, 再将它们代人各个磁亚点阵的 Brillouin 函数, 略去分 子场系数随温度的变化, 联立解出各磁亚点阵的磁矩以及它们的合磁矩随温度的变化(图 2 中 圆圈). 可以看出, 理论与实验相差较大. 但若采用 Dionne 提出的关于 YIG 中分子场系数 $\lambda_{a d}, \lambda_{d d}$ 和 $\lambda_{a d}$ 随非磁性离子代换分数 $k_{d}$ 和 $k_{a}$ 变化的经验公式 $\left(k_{a} \leqslant 0.35, k_{d} \leqslant 0.65\right)$ : $\lambda_{a d}=97.0\left(1-0.38 k_{d}-0.25 k_{a}\right), \lambda_{d d}=-30.4\left(1-0.87 k_{a}\right), \lambda_{a d}=-65.0\left(1-1.26 k_{d}\right)$. 
将它们代人各个磁亚点阵的 Brillouin 函数, 联立解出各磁亚点阵的磁矩及它们的合磁矩随温 度的变化(图 2 中虚线). 可以看出, 同绝对饱和磁矩的情况一样, 由 Diome 理论计算得到的 饱和磁矩随温度的变化曲线与实验结果(图 2 中实线)是符合得很好的.

因此,应用穆斯堡尔效应测定非磁性离子代换的铁氧体中磁亚点阵的磁性离子分布, 从而 计算饱和磁矩及其随温度的变化, 这对于铁氧体饱和磁化强度的分子设计是十分有用的. 同 时, 从实验结果分析以及同理论的比较可以看出, 在非磁性离子代换量较高的情况下, N\&el 的 简单亚铁磁性理论已经同实验有较大的差异, 只有正确地考虑了磁亚点阵间和磁亚点阵内的 反铁磁交换作用, 以及由后者引起的无规成角效应, 即采用 Dionne 的理论模型及其综合实验 结果得到的有关参数的经验公式, 才能得到与实验符合的结果. 有时还须对这些经验公式中 的系数作必要的修正.

拊录 1 此化学分析的详细结果为:

\begin{tabular}{c|c|c}
\hline 元素名称 & 本元美在样品中所占重量百分比 $(\%)$ & 相应于一个化学式中原子数 \\
\hline $\mathrm{Fe}$ & $30.00 \pm 0.15$ & $4.11 \pm 0.02$ \\
\hline $\mathrm{In}$ & $8.96 \pm 0.1$ & $0.595 \pm 0.007$ \\
\hline $\mathrm{Ge}$ & $2.38 \pm$ 小于 0.1 & $0.250 \pm$ 小于 0.01 \\
\hline $\mathrm{Ca}$ & $1.28 \pm 0.04$ & $0.245 \pm 0.008$ \\
\hline $\mathrm{Y}$ & $32.31 \pm 0.15 \%$ & $2.78 \pm 0.01$ \\
\hline
\end{tabular}

拊录 2 实际上 $\mathrm{Y}^{3+}$ 离子进入八面体晶位的结论是在综合分析对本样品所做的各种实验的结果（如： 从穆斯堡尔谱得到的 $\mathrm{Fe}^{3+}$ 离子分布, 晶格常数, 理论密度和实际密度的比及铁磁共振线宽等)并使之互相洽 合的基础上得出的 ${ }^{[1]}$. 本工作中用 $\mathrm{X}$ 射线测舅得到的晶格常数 $a_{0}=12.4608 \pm 0.0006 \AA$, 比文献 [11]中给出 的同样成分的样品的晶格常数 $a=12.4520 \pm 0.0006 \AA$ 显著地偏大，而且由于样品的共振线宽很小，故认 为多出来的这部分 $\mathbf{Y}^{3+}$ 不可能生成微量的钻铟石第二相,而只能是进人八面体 ${ }^{[1]}$.

致谢: 作者对物理研究所的郭照斌、王金玲同志, 高能物理研究所穆斯堡尔组的有关同志在测量工作中 的帮助, 对西南应用磁学研究所提供实验用样品, 表示深切感谢.

\section{考文献}

[1]林梅梅、本士、本国栋(将发表).

[2]本国栋、徐英庭,物理学报, 25(1976), 444.

[ 3 ] Czerlinsky, E., Phys. Stat. Solid., 34(1969), 484.

[4] N6el, L., Ann. de Phys., 3(1948), 137.

[5] Gilleo, M., J. Phys. Chem. Solids., 13(1960), 33.

[ 6 ] Geller, B., Bell. Sys. Tech. J., 43(1964), 565.

[. 7] Dionne, F., J. Appl. Phys., 41(1970), 4874.

[8] Wang, F. F. Y., in Treatise on Mat. Soi. Tech. (Ed. Herman, H.), 2(1973), 280.

[ 9] 本荫远、李国标, 铁样体物理学(修订本), 1978.

[10] Милль Б. В., Докл. Акад. Наук СССР, 165 (1965), 555.

[11] Winkler, G. et al., Philips Res. Repts., 27(1972), 151.

[12] Pauthenet, R., Ann. de Phys., 13(1958), 424. 